\title{
A special linear associative algebra
}

\author{
By J. H. M. WEDderburN.
}

(Received 4th February, 1938. Read 4th February, 1938.)

The following algebra possesses certain points of interest and is, I think, worth putting on record; it includes the algebra of matrices as a special case. Consider the algebra $H$ over a ring $F$ defined by

$$
h_{p q} h_{r s}=k_{q r} h_{p s}, \quad k_{q r}<F
$$

where $h_{p q}(p, q=1,2, \ldots, n)$ are linearly independent over $F$. If $a=\Sigma a_{i j} h_{i j}, b=\Sigma b_{i j} h_{i j}$ are any elements of $H$, then from (1)

$$
\begin{gathered}
a b=\sum_{p, q, r, s} a_{p q} b_{r s} k_{q r} h_{p s}=\Sigma c_{i j} h_{i j} \\
c_{i j}=\sum_{q r} a_{i q} k_{q r} b_{r j} .
\end{gathered}
$$

Hence, if we set $A=\left\|a_{i j}\right\|, B=\left\|b_{i j}\right\|, K=\left\|k_{i j}\right\|$ and consider the isomorphism $a \sim A$, we have $a+b \sim A+B$, $a b \sim A K B$; this shows, as is otherwise obvious, that $H$ is associative.

If $K$ has an inverse in $F$, we may define a set of elements $e_{p q}$ in $H$ by

which gives

$$
e_{p q} \sim K^{-1} E_{p q}, \quad E_{p q}=\left\|\delta_{i j}^{p q}\right\|
$$

$$
e_{p q} e_{r s} \sim K^{-1} E_{p q} K K^{-1} E_{r s}=K^{-1} E_{p q} E_{r s}=\delta_{q r} K^{-1} E_{p s} \sim \delta_{q r} e_{p s},
$$

so that $e_{p q}$ have the law of combination of ordinary matric units. Further the $e_{p q}$ are linearly independent since the $E_{p q}$ are and $K$ is non-singular; hence in this case $H$ is equivalent to the algebra of matrices.

Suppose now that $K^{-1}$ does not exist in $F$ but that the latter is restricted to be a Euclidean domain of integrity, that is, one in which the Euclidean algorism is valid. If we define a new basis for $H$ by

$$
\begin{aligned}
& h^{\prime}{ }_{1 q}=h_{1 q}+\theta h_{2 q}, \quad(q=1,2, \ldots, n), \quad \theta \text { in } F \\
& h^{\prime}{ }_{p q}=h_{p q}, \quad(p \neq 1),
\end{aligned}
$$

then these elements are linearly independent, and so in fact form a basis, and a short calculation shows that their law of combination is

$$
h_{p q}^{\prime} h_{r 8}^{\prime}= \begin{cases}\left(k_{q 1}+\theta k_{q 2}\right) h_{p 8}^{\prime} & (r=1) \\ k_{q r} h_{p s}^{\prime} & (r \neq 1) .\end{cases}
$$


Hence the transformation (4) gives rise to the corresponding elementary transformation on the columns of $K$. Interchanging the rôles of the subscripts clearly gives the same transformation on the rows of $K$; and similar results apply to permutations of one set of subscripts. Hence, since $F$ is Euclidean, we may assume $K$ to be in its normal form, that is, such that if its rank is $r$, the first $r$ coefficients in the main diagonal are $k_{1}, k_{2}, \ldots, k_{r}$ with $k_{i} \mid k_{i+1}(i=1,2, \ldots, r-1)$ and all other coefficients are 0 . If the rank is $n$, this form differs from a matric algebra only in this that $k_{s 8}$ is not necessarily unity so that the algebra is the matric algebra when $F$ is a field. If $r<n$, the subalgebra $H_{1}:\left(h_{p q}, p, q=1,2, \ldots, r\right)$ is simple and when $F$ is a field is a simple matric algebra; and

$$
\begin{array}{r}
N:\left(h_{p q}, p=r+1, \ldots, n ; q=1,2, \ldots, n\right. \\
\quad \text { or } p=1,2, \ldots, r ; q=r+1, \ldots, n)
\end{array}
$$

is the radical since in $h_{p q} h_{i j}=k_{q i} h_{p j}$ we have $k_{q i}=0$ if $p, q \leqq r$ and $i>r$ while, if $j>r$, then $h_{p j}<N$. The difference algebra $(H-N)$ is, of course, isomorphic with $H_{1}$.

It is of some interest to observe that when $F$ is a field and no $k_{p q}$ is zero, which can always be secured by elementary transformations, then the basis defined by

$$
f_{p q}=h_{p q} / k_{q p}
$$

is composed entirely of idempotent elements. An example of this has already been given. ${ }^{1}$

${ }^{1}$ Question 3700, Amer. Math. Monthly, 41 (1934), p. 521 and 43 (1936), p. 378.

Princeton University, Princeton, N.J. 